\title{
Optimal management of urinary tract infections in older people
}

This article was published in the following Dove Press journal:

Clinical Interventions in Aging

21 June 20II

Number of times this article has been viewed

\author{
Louise A Beveridge' \\ Peter G Davey ${ }^{2}$ \\ Gabby Phillips ${ }^{3}$ \\ Marion ET McMurdo' \\ 'Ageing and Health, Division \\ of Medical Sciences, Ninewells \\ Hospital and Medical School, ${ }^{2} \mathrm{Health}$ \\ Informatics Centre, University of \\ Dundee, ${ }^{3}$ Medical Microbiology \\ Department, NHS Tayside
}

\begin{abstract}
Urinary tract infections (UTI) occur frequently in older people. Unfortunately, UTI is commonly overdiagnosed and overtreated on the basis of nonspecific clinical signs and symptoms. The diagnosis of a UTI in the older patient requires the presence of new urinary symptoms, with or without systemic symptoms. Urinalysis is commonly used to diagnose infection in this population, however, the evidence for its use is limited. There is overwhelming evidence that asymptomatic bacteriuria should not be treated. Catheter associated urinary tract infection accounts for a significant amount of hospital-associated infection. Indwelling urinary catheters should be avoided where possible and alternatives sought. The use of narrow spectrum antimicrobial agents for urinary tract infection is advocated. Local guidelines are now widely used to reflect local resistance patterns and available agents. Guidelines need to be updated to reflect changes in antimicrobial prescribing and a move from broad to narrow spectrum antimicrobials.
\end{abstract}

Keywords: urinary tract infection, elderly, review

\section{Introduction}

Urinary tract infections (UTI) are one of the most common infections in the older population, occurring both in the community and in long-term care settings. The spectrum of UTI ranges from symptomatic bacteriuria to bacteremic infection. UTI with bacteremia has a high mortality in the older population, with studies reporting a 28 day mortality of $5 \% .^{1}$

Too often, the diagnosis of UTI is made in the absence of a typical clinical history and signs resulting in overdiagnosis and overtreatment. ${ }^{2}$ Studies suggest that UTI is incorrectly diagnosed in as many as $40 \%$ of hospitalized older people. ${ }^{3}$ The increasing prevalence of health care associated infection such as Clostridium difficile and emerging antibiotic resistance highlights the importance of obtaining a firm diagnosis, treating with appropriate antibiotics and avoiding the use of broad spectrum antibiotics. ${ }^{4}$

There is a paucity of evidence-based guidelines for the management of UTI specifically in an older population. The aim of this article is to review the current evidence on the diagnosis, management, and prevention of UTI in older people.

\section{Epidemiology}

Case definitions are central to any discussion about the epidemiology of UTI (Table 1). Symptomatic UTI requires the presence of new urinary tract symptoms such as frequency, urgency, dysuria, new incontinence, or costovertebral or suprapubic tenderness. The diagnosis of UTI is actually very difficult because there are no 
Table I Definition of terms

\begin{tabular}{|c|c|c|}
\hline Term & Standard definition & $\begin{array}{l}\text { Application to management of urinary tract infection (UTI) } \\
\text { in elderly patients }\end{array}$ \\
\hline $\begin{array}{l}\text { Significant } \\
\text { bacteriuria }\end{array}$ & $\begin{array}{l}\text { Presence of a specified number } \\
\text { (usually }>10^{5} \mathrm{CFU} / \mathrm{mL} \text { ) of a single } \\
\text { species of bacteria in a freshly voided, } \\
\text { midstream specimen of urine. }\end{array}$ & $\begin{array}{l}\text { This level of bacteriuria is significant because it is unlikely that it can } \\
\text { be explained by contamination of the urine sample with perineal flora. } \\
\text { It DOES NOT indicate clinically significant bacteriuria. "Significant" } \\
\text { bacteriuria is often asymptomatic and does not necessarily require } \\
\text { treatment. Lower levels may sometimes be significant. }\end{array}$ \\
\hline $\begin{array}{l}\text { Uncomplicated } \\
\text { urinary tract } \\
\text { infection }\end{array}$ & $\begin{array}{l}\text { Lower urinary tract infection in an adult } \\
\text { woman who is not pregnant and has no } \\
\text { underlying abnormality of the urinary tract } \\
\text { or indwelling urinary device. }\end{array}$ & $\begin{array}{l}\text { The evidence about effectiveness of short ( } 3 \text { day) courses of treatment } \\
\text { for UTI only applies to uncomplicated UTIs. All UTIs in males, all UTIs } \\
\text { associated with urinary catheters, and all UTIs with systemic symptoms } \\
\text { are complicated UTIs. }\end{array}$ \\
\hline $\begin{array}{l}\text { Lower urinary } \\
\text { tract infection }\end{array}$ & $\begin{array}{l}\text { Infection confined to the tissues of the } \\
\text { bladder or urethra. The presence of } \\
\text { symptoms or signs of systemic infection } \\
\text { indicates upper urinary tract infection. }\end{array}$ & $\begin{array}{l}\text { Both nitrofurantoin and fosfomycin only achieve effective } \\
\text { concentrations in the lower urinary tract infection. These antibiotics } \\
\text { should not be used to treat patients with systemic symptoms or signs. }\end{array}$ \\
\hline
\end{tabular}

currently available diagnostic techniques for demonstrating that bacteria have invaded the tissues of the urinary tract. The diagnosis is therefore clinical and is based on symptoms or signs of inflammation of the urinary tract. Urine culture does not establish the diagnosis of UTI but does aid in the selection of appropriate treatment.

A number of factors predispose older patients to UTI. Use of urinary catheters and external urine collection devices increase the frequency of bacteriuria. Asymptomatic bacteriuria is more common in patients with cognitive impairment and urinary and fecal incontinence. Neurological conditions such as cerebrovascular disease, Alzheimer's disease, and Parkinson's disease are all common in later life and are associated with impaired bladder emptying. ${ }^{5,6}$ Patients with diabetes mellitus also have increased prevalence of asymptomatic bacteriuria with contributing factors including neurogenic bladder and poor glycemic control. ${ }^{7}$

In women, postmenopausal estrogen deficiency has been linked with recurrent UTI. ${ }^{8}$ Cystoceles, significant postvoiding residual urine volumes, and incontinence are all associated with recurrent UTI. ${ }^{8}$ In men, prostatic disease leads to urinary symptoms and urinary retention. Any urological condition that can cause obstruction such as stones or tumor also increases the risk of urinary infection. Urinary tract infection most commonly arises from ascending infection via the urethra with Gram-negative organisms in the perineal region from bowel flora. ${ }^{9}$

Bacteriuria is defined as the presence of bacteria in urine on microscopy or quantitative culture. It is important to appreciate that the presence of bacteriuria does not always represent disease. ${ }^{10}$ Pyuria represents the presence of white blood cells in urine. White blood cells may be present without bacteriuria and are particularly associated with the presence of a urinary catheter, stone, tumor, or infection of the lower genital tract. ${ }^{10}$ Urinary tract infections are subclassified into complicated and uncomplicated UTI. Complicated UTI implies that the urinary tract has a functional or structural abnormality but the term also includes all upper UTI and all UTI in men (Table 1). Older female patients frequently have functional or structural abnormalities of the urinary tract and many regard UTI as complicated UTI in this group. ${ }^{11}$

\section{Diagnosis \\ Clinical findings}

The diagnosis of infection in an older patient is often complicated by the lack of typical symptoms and a clear history. Up to one-third of elderly patients do not present pyrexia in response to infection. ${ }^{12}$ The presence of cognitive impairment and communication difficulties can make it difficult to obtain an accurate history. Many older patients have chronic genitourinary symptoms and it is important to recognize that this is not synonymous with infection. ${ }^{8}$

Many clinicians incorrectly attribute factors such as functional decline, increased confusion, and nonspecific signs and symptoms to urinary tract infections and start treatment on this basis. ${ }^{2,13,14}$ In the absence of clear urinary tract localizing symptoms and signs, older patients should be systematically assessed with a full clinical evaluation performed to arrive at a differential diagnosis. ${ }^{2}$

Juthani-Mehta et al conducted a prospective cohort study of nursing home residents in Connecticut to ascertain which clinical features are associated with bacteriuria and pyuria in uncatheterized nursing home residents. ${ }^{15}$ Dysuria, change in character of urine and change in mental status were significantly associated with bacteriuria plus pyuria in patients with suspected UTI. Absence of these clinical 
features identified residents at low risk of having bacteriuria plus pyuria $(25.5 \%)$, whereas the presence of dysuria plus one or both of the other clinical features identified residents at high risk of having bacteriuria plus pyuria (63.2\%). Participants had a high prevalence of dementia (63.3\%) and given the high prevalence of bacteriuria in patients with cognitive impairment, altered mental status may not be a useful criterion for commencing antibiotics. This study reinforced that nonspecific signs, such as change in function, malaise, falls, and change in voiding pattern were not significantly associated with bacteriura plus pyuria.

Recognizing the diagnostic challenges in this population, Loeb et al attempted to develop criteria for initiating antibiotics in patients with bacteriuria in a long-term care setting. ${ }^{16}$ This consensus paper suggested that antibiotics should be initiated for residents without a urinary catheter with acute dysuria alone or fever (defined as temperature above $37.9^{\circ} \mathrm{C}$ or rise of $1.5^{\circ} \mathrm{C}$ above baseline) and one of the following symptoms: new or worsening urgency, frequency, suprapubic pain, frank hematuria, costovertebral angle tenderness, or urinary incontinence.

Some older patients may have UTI without localizing urinary symptoms and these patients cannot always be identified. The approach suggested by Nicolle is simply to closely monitor such patients and regularly reassess clinical features. ${ }^{14}$

\section{Urine dipstick testing}

Few studies have looked specifically at the role of dipstick urinalysis in the older population. Most studies on urine dipstick testing discuss the sensitivity and specificity of the tests. ${ }^{17}$ While these characteristics are relevant to the performance of tests in a population, they are rarely helpful for making decisions about individual patients. ${ }^{18}$ In contrast, the positive predictive value (PPV) and negative predictive value (NPV) of a test are helpful in estimating the probability of disease in patients with positive and negative tests. Bent et al used likelihood ratios to express the post-test probability of a female patient having an uncomplicated UTI. ${ }^{19}$ In a systematic review based on studies in young women, nitrite and leucocyte esterase positive urinalysis had a positive likelihood ratio of 4.2. A negative urinalysis result had a likelihood ratio of UTI of 0.3 . However this review was not specific to older people so the conclusions may not be transferable.

Sundvall and Gunnarsson evaluated the NPV of combined nitrite and leucocyte esterase dipstick analysis in predicting the presence of pathogenic bacteria in elderly nursing home patients. ${ }^{20}$ They addressed shortcomings in previous studies by including both visual and analyzer reading of dipsticks as well as providing data about the separate performance of tests for nitrites and leukocytes in the identification of specific bacteria in addition to any bacteria. However, an important weakness of the study is its failure to provide separate data for symptomatic versus asymptomatic patients, and the case definition for significant bacteriuria implies that symptomatic patients were included.

According to their statistical analysis, ruling in or ruling out bacteriuria was considered possible where the point estimate of PPV/NPV was $\geq 85 \%$ with a lower confidence interval (CI) of $\geq 80 \%$. The authors concluded that the most reliable way to rule out bacteriuria was to identify patients in whom both tests were negative. However, the authors concede that no combination of dipstick test results can be used to rule in bacteriuria. The combination of negative leukocyte and nitrite test had a NPV of 88\% (CI: 84\%-92\%), however the results show interpretation is further influenced by the definition of a negative test because the PPV is only $51 \%$ if a positive test is defined as $>0$ (Figure 1). Our interpretation of these results is that dipstick testing is likely to add little if anything to clinical diagnosis. With a positive test, the probability of bacteriuria was between $51 \%$ and $73 \%$, and with a negative test it was between $9 \%$ and $21 \%$. These results are similar to the positive and negative predictive value of clinical symptoms and signs without stick testing.

In the study by Juthani-Mehta et al, dysuria - change in character of urine - and change in mental status were significantly associated with the combined outcome of bacteriuria plus pyuria. ${ }^{15}$ Absence of these clinical features identified residents at low risk of having bacteriuria plus pyuria $(25.5 \%)$, whereas the presence of dysuria plus one or both of the other clinical features identified residents at high risk of having bacteriuria plus pyuria (63.2\%). Supporters of dipstick testing need to provide evidence that these tests meaningfully improve clinical prediction of bacteriuria in symptomatic patients.

\section{Urine culture}

Significant bacteriuria is commonly defined as greater than $10^{5}$ colony forming units $/ \mathrm{mL}$ of a single bacterial species in a freshly voided sample of urine. ${ }^{10}$ However, case definitions in studies of UTI are often much more complex, for example:

"A culture with growth of potentially pathogenic bacteria was normally considered positive if the number of colony forming units per liter (CFU/mL) was $>10^{5}$. In case of specific signs of possible UTI such as positive nitrite dipstick, 


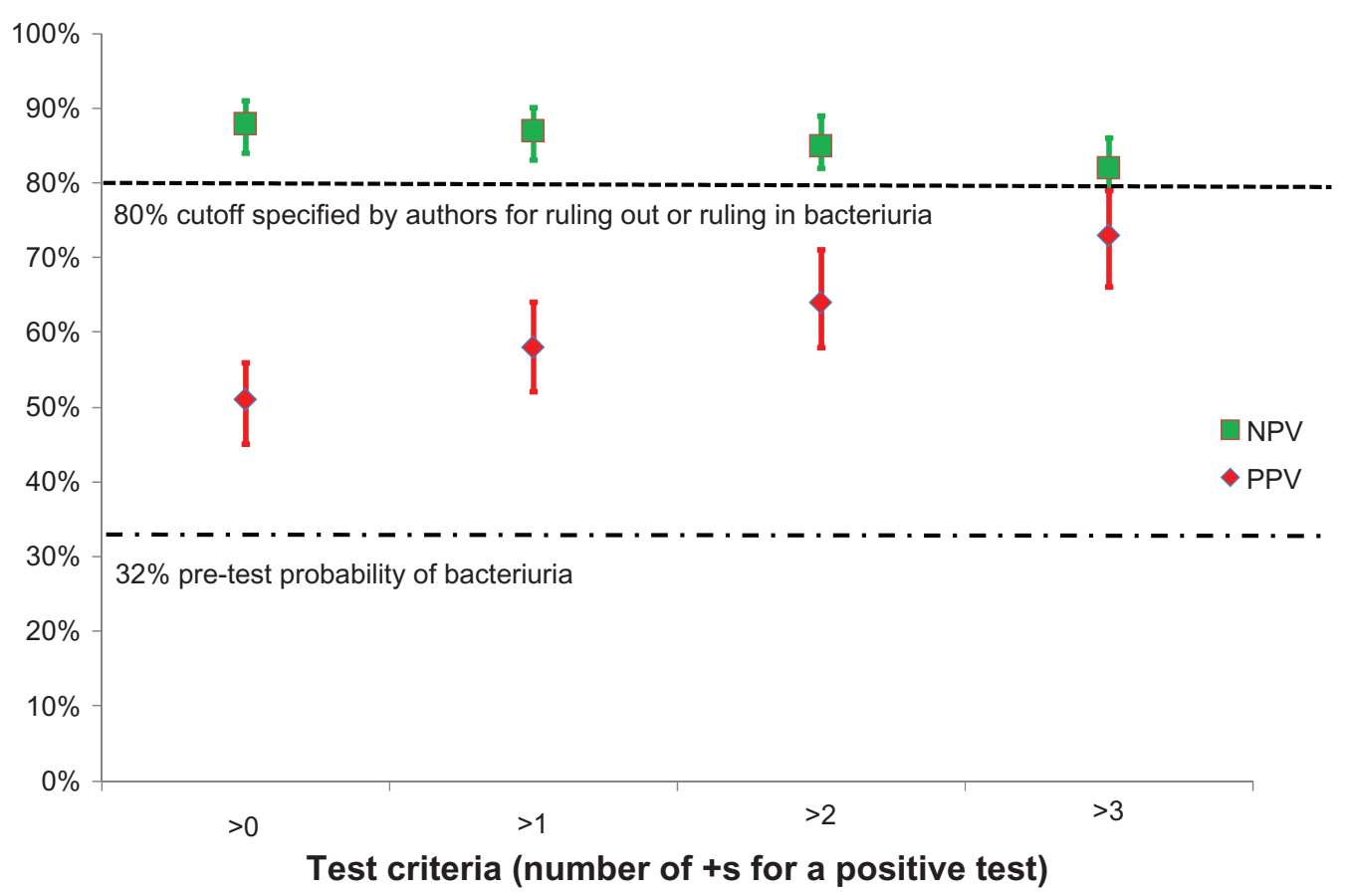

Figure I Negative predictive value (NPV) and positive predictive value (PPV) for dipstick urine testing for diagnosis of bacteriuria in a nursing home population. Test characteristics of a positive leukocyte esterase and/or a positive nitrite dipstick were compared to urine culture. These results were for visual reading of the dipstick; results with analyzer reading were almost identical. Data adapted from Sundvall and Gunnarsson. ${ }^{20}$

leukocyte esterase dipstick $>1$, fever, frequency, urgency or dysuria, the cut-off point was $>10^{3}$ for patients harbouring Escherichia coli and male patients with Klebsiella species and Enterococcus faecalis. For symptomatic women with the two latter species, the cut-off level was $>10^{4}$

\section{Sundvall and Gunnarsson ${ }^{20}$}

Obtaining urine cultures can be difficult in some older people. Guidelines suggest that an in-and-out catheter may be the most reliable way of obtaining a urine sample in the female population if a voided sample cannot be obtained. ${ }^{21}$ Urine culture by needle aspiration of the bladder is the gold standard method for diagnosing bacteriuria, but is rarely used in older people. ${ }^{10}$ Urine cultures should not be sent in individuals who are asymptomatic. ${ }^{10,21}$ Urine should also not be sent for culture on the basis of positive urine dipsticks if no symptoms are evident. ${ }^{22}$

\section{Asymptomatic bacteriuria}

Asymptomatic bacteriuria is defined as the presence of bacteria in urine on microscopy or quantitative culture in a specimen obtained from a patient who does not have typical symptoms of a urinary tract infection. ${ }^{10}$ This requires confirmation by two consecutive samples. Studies have suggested a prevalence rate of $25 \%-50 \%$ of women and $15 \%-40 \%$ of men without catheters amongst nursing home residents. ${ }^{22}$
Risk factors associated with asymptomatic bacteriuria include institutionalization, presence of a urinary catheter, female sex, increasing age, and diabetes. ${ }^{10}$

It is widely recognized that asymptomatic bacteriuria should not be treated with antibiotics in the elderly population. ${ }^{10,22}$ Treating asymptomatic bacteriuria does not reduce mortality and can cause harm. ${ }^{23,24}$ Guidelines suggest that for every three people treated with antibiotics, one will come to harm (CI: 2-10). ${ }^{10}$ Evidence also suggests that treating asymptomatic bacteriuria in nursing home patients who have chronic stable incontinence does not improve incontinence in the short-term. ${ }^{25}$

\section{Catheter-associated UTI}

Catheter-associated UTIs account for a high proportion of health care associated infection and are common among the older population in long-term care. In ambulatory patients, single catheter insertion is associated with urinary tract infection in $1 \%-2 \%$ of patients. Indwelling urinary catheters lead to almost universal bacteriuria within 3-4 days of catheterization. Catheterization allows for the formation of a biofilm between the catheter and urethral mucosa. A biofilm is the aggregation of microorganisms that form a structure on solid surfaces. ${ }^{26,27}$ The greatest risk factor for catheter-associated UTI is duration of catheterization. $^{8}$ 
The diagnosis of catheter associated UTI in the older population is difficult and clinical signs and symptoms are often absent. Guidelines suggest symptoms or signs alone should not be relied upon for predicting the likelihood of catheter associated UTI. ${ }^{10}$ Catheter samples of urine should only be sent if the patient shows signs of sepsis, recognizing that most catheterized patients will have bacteriuria. ${ }^{10,22}$ Urinary catheters should ideally be removed and a culture then acquired from a freshly inserted catheter prior to commencing antimicrobials. ${ }^{26}$

Loeb et al recommended minimum criteria for initiating antibiotics in older patients in long-term care with catheters. ${ }^{16}$ These criteria included presence of fever $>37.9^{\circ} \mathrm{C}$ or $1.5^{\circ} \mathrm{C}$ above baseline temperature, new costovertebral tenderness, rigors without obvious cause, or new onset of delirium. SIGN (Scottish Intercollegiate Guidelines Network) guidelines advocate that in patients with indwelling urinary catheters who are pyrexial, the clinician should exclude other sources of infection and look for any localizing signs such as suprapubic tenderness or loin pain. ${ }^{10}$ A sample of urine should be cultured to isolate the infective organism and to obtain antibiotic sensitivities stating clearly on the request form that it is a sample of catheter urine. The severity of infection and underlying comorbidities should be taken into consideration prior to prescribing antibiotics.

The Infectious Diseases Society of America (IDSA) published international clinical practice guidelines in 2009 on the diagnosis and management of catheter-associated UTI. ${ }^{26}$ These apply to adults over 18 years of age and are not specific to older people. Similar clinical criteria to Loeb's were identified for diagnosis of catheter associated UTI. New or worsening fever, rigors, altered mental state, general malaise, or lethargy without other identified causes were identified as possible signs and symptoms.

Guidelines suggest that catheters should be replaced prior to commencing antibiotic therapy in symptomatic catheterized patients if it has been in situ for longer than 1 week. . $^{10,22,26}$ IDSA guidelines recommend that catheters that have been in use for more than 2 weeks should be changed to try and lead to quicker resolution of symptoms and prevent UTI recurrence. ${ }^{26}$ The optimal method of decreasing catheter associated UTI is to reduce indwelling catheter use and remove catheters the moment they are no longer clinically necessary.

\section{Management of UTI Antimicrobial prescribing}

In an era of antibiotic stewardship, the prescribing of antibiotics is the subject of much scrutiny. ${ }^{28}$ The trend until recently of increasing cases of Clostridium difficile diarrhea has led to a shift from broad to narrow spectrum antibiotics. ${ }^{4}$ The prevalence of trimethoprim resistance in the Tayside population in Scotland has been reported as $27 \%-28 \% .{ }^{29}$ Given the increased problems with resistance and health care associated infection, the use of narrow spectrum agents where possible is now strongly encouraged. ${ }^{30}$ The use of narrow spectrum agents highlights the necessity for accurate diagnosis and the importance of obtaining cultures in the elderly prior to commencing antibiotics. ${ }^{31}$ Local guidelines are now widely utilized for prescribing antimicrobials based on local resistance patterns and available agents.

The most common organism isolated depends on populations studied. E. coli is the most frequent organism isolated in the older population. ${ }^{1,11,32}$ Gram-negative agents such as Klebsiella pneumoniae and Proteus mirabilis are also common.

The management of uncomplicated symptomatic UTI in the female population has been the subject of several randomized controlled trials. Most studies exclude the very elderly and focus on the younger adult population. UK guidelines advocate that trimethoprim should be used as the first-line antibiotic in uncomplicated symptomatic UTI in females. ${ }^{10,23,30}$ Nitrofurantoin is an alternative, although it should not be used in those with renal impairment due to the inability to achieve necessary concentrations in the urine and possibility of toxic levels in the plasma. ${ }^{10}$ European guidelines are now moving towards fosfomycin trometamol or nitrofurantoin as first- and second-line agents with trimethoprim or cotrimoxazole advised only in areas where resistance rates for E. coli are less than $20 \%{ }^{8}$

The Infectious Disease Society of America published guidelines on the management of UTIs in females, which are currently under review. ${ }^{33}$ Trimethoprim-sulfamethoxazole was advocated as the first-line agent for acute symptomatic lower urinary tract infection in females for 3 days. Trimethoprim and ofloxacin were proposed as suitable alternatives. Fluoroquinolones were not recommended as firstline therapy unless there was a high level $(>10 \%-20 \%)$ of trimethoprim or trimethoprim-sulfamethoxazole resistance. They also identified that nitrofurantoin and fosfomycin use may be more prominent if increasing resistance emerged. With growing concern over fluoroquinolone use and resistance, a group reviewed the recommendations for empiric treatment of UTI. ${ }^{34}$ They agreed that trimethoprim-sulfamethoxazole should continue to be used as first-line treatment in uncomplicated UTI if the patient has not received any antibiotics in the past 3 months, had no hospitalizations, and there is $<20 \%$ 
resistance to trimethoprim-sulfamethoxazole locally. They recommended considering nitrofurantoin or fosfomycin if these criteria were not fulfilled. Fluoroquinolones were recommended only if severe symptoms were present and the patient had received previous antibiotic therapy in the last 3 months and lived in an area with $>20 \%$ resistance to trimethoprim-sulfamethoxazole. These guidelines were not specific to the older population.

A 3-day course of trimethoprim is recommended for women and a 7-day course for men. ${ }^{22}$ A Cochrane review examined evidence for duration of antibiotic therapy for uncomplicated, symptomatic lower UTIs in older women. ${ }^{35}$ Fifteen randomized controlled studies (1644 elderly females) were reviewed and the authors concluded that short course antibiotics of 3-6 days could be adequate for treating uncomplicated UTI in older women. A need for more studies looking at optimum duration of narrow spectrum agents was highlighted.

Most published guidelines for management of upper UTIs still recommend using quinolones as first-line agents. ${ }^{10}$ The need to review such guidelines has been highlighted. ${ }^{4}$ The IDSA guidelines recognized that there were few good, recent clinical studies of treatment in pyelonephritis and upper UTIs. ${ }^{33}$ They advised that severe pyelonephritis should be treated with intravenous fluoroquinolones or an aminoglycoside with or without ampicillin or an extended spectrum cephalosporin with or without an aminoglycoside. These guidelines again were not specific to the elderly population.

Our local guidelines in Tayside, Scotland advocate that lower UTIs in females should be managed with trimethoprim for 3 days and males should receive 14 days of trimethoprim. Complicated UTIs, including upper UTI and bacteremic UTI, are treated with intravenous amoxicillin and gentamicin. Co-trimoxazole is used as an alternative in penicillin allergic patients in conjunction with gentamicin.

Problems with antimicrobial resistance can also be tackled with more judicious use of antibiotics. Research has sought to uncover why doctors treat asymptomatic bacteriuria and also to identify ways of reducing antimicrobial prescribing in the older population. ${ }^{13,36}$ Loeb et al conducted a cluster randomized controlled trial to assess whether the use of an algorithm to aid diagnosis and management of urinary tract infections could reduce antimicrobial prescribing in nursing home residents in Canada. ${ }^{36}$ This used educational material aimed at nurses and physicians working in nursing homes and set out a diagnostic algorithm based on previous consensus criteria for diagnosing UTIs in catheterized and noncatheterized long-term care patients. ${ }^{16}$ The study was underpowered to assess significant differences in hospital admission and death between the group randomized to intervention and the usual care group. It did however demonstrate that using an algorithmic approach reduced antimicrobial prescribing for suspected UTI. ${ }^{36}$

If patients do not have systemic signs there is no need to start antibiotics immediately. In a randomized trial of five management strategies for uncomplicated UTI in nonpregnant women delaying antibiotics to see if symptoms resolved spontaneously did not increase duration or severity of symptoms and significantly reduced antibiotic use. Symptom score was 2.11 for delayed antibiotics vs 2.15 for immediate antibiotics but there was a $20 \%$ reduction in antibiotic use. This trial was done in younger women (mean age 40) but the results are relevant to older women, especially in care homes where they can be observed closely. ${ }^{37}$

\section{Prevention of UTI Cranberry products}

Cranberries have long been considered to have a role in reducing UTIs. The exact mechanism has been disputed. Cranberries contain tannins called proanthocyanidins, which prevent adherence of P-fimbriated E. coli to uroepithelial cells on the bladder wall. ${ }^{38}$

A Cochrane review examined the evidence for the use of cranberry in the prevention of UTI in susceptible populations. ${ }^{39}$ This identified ten randomized controlled trials using cranberry for the prevention of UTI. Four studies were included in the meta-analysis and demonstrated that cranberry significantly reduced the incidence of UTI at 1 year compared to placebo. Studies included a variety of populations examined and cranberry was more effective in younger adult females with recurrent UTI than in older people.

McMurdo et al conducted a randomized, placebo-controlled double-blind trial examining whether cranberry juice reduced UTI in elderly hospitalized patients in Scotland. ${ }^{40}$ The primary outcome was time to onset of first symptomatic UTI. 376 patients were randomized making this one of the largest available randomized controls examining the effect of cranberry juice. This study had a lower symptomatic infection rate than that observed during the pilot phase and so was underpowered to demonstrate a significant difference between cranberry and placebo. The number of $E$. coli UTI was significantly lower in the cranberry group compared to placebo.

SIGN guidelines recommended that females with recurrent UTI should take cranberry products to decrease 
frequency of recurrence. ${ }^{10}$ Capsules were advocated as easier to take than juice. Cranberry products are not recommended for patients taking warfarin as cranberry is thought to potentiate the effects of warfarin. The United Kingdom Committee on Safety of Medicines issued an alert in 2004 about this based on a series of case reports. ${ }^{41}$ However, subsequent work suggests that there is no significant interaction between warfarin and high-dose cranberry juice. ${ }^{42}$

\section{Prevention of catheter associated UTI}

Catheter associated UTI is the most common health care associated infection throughout the world and is common in long-term care facilities. Urinary catheterization should be avoided unless there is a clear clinical indication. Catheters should be avoided where possible for the management of incontinence. Staff should also be trained on indications for catheterization and written protocols should be put in place. ${ }^{26,27}$ Catheters should also be removed the moment that they are no longer required.

Alternatives to indwelling urethral catheters should be considered. Condom catheters are associated with a lower incidence of bacteriuria, however their use is sometimes difficult in confused patients. ${ }^{27} \mathrm{~A}$ Cochrane review on shortterm urinary catheterization in adults found that suprapubic catheterization was associated with less bacteriuria than urethral catheterization. ${ }^{43}$ Suprapubic catheterization does carry a small risk of visceral injury on insertion through the abdominal wall. Intermittent catheterization was also associated with a lower risk of bacteriuria when compared to indwelling catheterization in this review, however studies included were mainly in an elective orthopedic setting. ${ }^{43}$

Guidelines suggest that antibiotic prophylaxis should not be used to prevent catheter associated UTI in catheterized patients. ${ }^{10}$ Although prophylaxis may decrease the incidence of asymptomatic bacteriuria in catheterized patients, it increases the risk of antimicrobial resistance.

\section{Conclusion}

Urinary tract infections present a significant problem in both hospital medicine and general practice. The burden of infection is high and there are many challenges in diagnosis and management. Several studies have identified the need for better education on asymptomatic bacteriuria and presentation of UTI in the elderly population. Symptomatic infections are often difficult to diagnose in older populations where there are high levels of cognitive impairment and communication difficulties, such as amongst residents of institutional care.
A strategy of observing patients for possible symptoms before initiating antibiotics have been advocated, although recognizing this may not always be practical in a primary care setting. Reducing inappropriate prescribing of antibiotics for asymptomatic bacteriuria is likely to reduce antimicrobial resistance.

With emerging knowledge on antibiotic resistance and health care-associated infection, guidelines need to be updated to reflect the need to prescribe narrow spectrum agents when available and avoid empirical use of broad spectrum antibiotics.

\section{Key points}

- Urinary tract infections are overdiagnosed and overtreated in older people.

- Diagnosis of UTI in an older person requires the presence of new urinary symptoms.

- Asymptomatic bacteriuria is very common in later life and should not be screened for or treated.

- Local guidelines and antibiotic policies should be used for management of UTI.

- Narrow spectrum antibiotics are generally preferable to broad spectrum agents.

\section{Disclosure}

No conflicts of interest were declared in relation to this paper.

\section{References}

1. Tal S, Guller V, Levi S, et al. Profile and prognosis of febrile elderly patients with bacteremic urinary tract infections. J Infect. 2005;50:296-305.

2. McMurdo MET, Gillespie ND. Urinary tract infection in old age: over-diagnosed and over-treated. Age Ageing. 2000;29:297-298.

3. Woodford HJ, George J. Diagnosis and management of urinary tract infection in hospitalized older people. J Am Geriatr Soc. 2009;57: 107-114.

4. Rao GG, Patel M. Urinary tract infection in hospitalized elderly patients in the United Kingdom: the importance of making an accurate diagnosis in the post broad-spectrum antibiotic era. J Antimicrob Chemother 2009;63:5-6.

5. Nicolle LE. Urinary tract infection in geriatric and institutionalised patients. Curr Opin Urol. 2002;12:51-55.

6. Nicolle LE. Urinary tract infection in long-term-care facility residents. Clin Infect Dis. 2000;31:757-761.

7. Zhanel GG, Nicolle LE, Harding GKM, et al. Prevalence of asymptomatic bacteriuria and associated host factors in women with diabetes mellitus. Clin Infect Dis. 1995;21:316-322.

8. Stamm WE, Raz P. Factors contributing to susceptibility of postmenopausal women to recurrent urinary tract infections. Clin Infect Dis. $1999 ; 28: 723-725$.

9. Grabe M, Bjerklund-Johansen TE, Botto H, et al. Guidelines on urological infections. European Association of Urology 2010. Available at: http://www.uroweb.org/gls/pdf/Urological\%20Infections\%202010. pdf. Accessed May 3, 2011.

10. Scottish Intercollegiate Guidelines Network [SIGN]. Management of suspected bacterial urinary tract infection in adults: a national clinical guideline. SIGN 2006. Available at: www.sign.ac.uk. Accessed May 3, 2011. 
11. Nicolle LE. Urinary tract pathogens in complicated infection and in elderly individuals. $J$ Infect Dis. 2001;183(Suppl 1):S5-S8.

12. Liang SY, Mackowiak PA. Infetcions in the elderly. Clin Geriatr Med. 2007;23:441-456.

13. Walker S, McGeer A, Simor AE, et al. Why are antibiotics prescribed for asymptomatic bacteriuria in institutionalised elderly people? A qualitative study of physicians' and nurses' perceptions. CMAJ. 2000;163: 273-277.

14. Nicolle LE. Asymptomatic bacteriuria in institutionalized elderly people: evidence and practice. CMAJ. 2000;163:285-286.

15. Juthani-Mehta M, Quagliarello V, Perrelli E, et al. Clinical features to identify urinary tract infections in nursing home residents: a Cohort study. J Am Geriatr Soc. 2009;57:963-970.

16. Loeb M, Bentley DW, Bradley S, et al. Development of minimum criteria for the initiation of antibiotics in residents of long-term-care facilities: results of a consensus conference. Infect Control Hosp Epidemiol. 2001;22:120-124.

17. Deville WL, Yzermans JC, van Duijn NP, et al. The urine dipstick test useful to rule out infections. A meta-analysis of the accuracy. $B M C$ Urol. 2004;4:4.

18. Jaeschke R, Guyatt GH, Sackett DL. Users' guides to the medical literature. III. How to use an article about a diagnostic test. B. What are the results and will they help me in caring for my patients? The Evidence-Based Medicine Working Group. JAMA. 1994;271: 703-707.

19. Bent S, Nallamothu BK, Simel DL, et al. Does this woman have an acute uncomplicated urinary tract infection? JAMA. 2002;287:2701-2710.

20. Sundvall P-D, Gunnarsson RK. Evaluation of dipstick analysis among elderly residents to detect bacteriuria: a cross-sectional study in 32 nursing homes. BMC Geriatr. 2009;9:32.

21. High KP, Bradley SF, Gravenstein S, et al. Clinical practice guideline for the evaluation of fever and infection in older adult residents of longterm care facilities: 2008 Update by the Infectious Diseases Society of America. Clin Infect Dis. 2009;48:149-171.

22. HPA. Diagnosis of UTI. Quick reference guide for primary care. 2009. Available at: http://www.hpa.org.uk/web/HPAwebFile/ HPAweb_C/1194947330877. Accessed May 3, 2011.

23. Nicolle LE. Urinary infections in the elderly: symptomatic or asymptomatic? Int J Antimicrob Agents. 1999;11:265-268.

24. Abrutyn E, Mossey J, Berlin JA, et al. Does asymptomatic bacteriuria predict mortality and does antimicrobial treatment reduce mortality in elderly ambulatory women? Ann Intern Med. 1994;120:827-833.

25. Ouslander JG, Schapira M, Schnelle JF, et al. Does eradicating bacteriuria affect the severity of chronic urinary incontinence in nursing home residents? Ann Intern Med. 1995;122:749-754.

26. Hooton TM, Bradley SF, Cardenas DD, et al. Diagnosis, prevention, and treatment of catheter-associated urinary tract infection in adults: 2009 International Clinical Practice Guidelines from the Infectious Diseases Society of America. Clin Infect Dis. 2010;50:625-663.

27. Tenke P, Kovacs B, Bjerklund Johansen TE, et al. European and Asian guidelines on management and prevention of catheter-associated urinary tract infections. Int J Antimicrob Agents. 2008;31(Suppl 1):S68-S78.

28. Dellit HT, Owens RC, McGowan GE, et al. Infectious Diseases Society of America and the Society for Healthcare Epidemiology of America guidelines for developing an institutional program to enhance antibiotic stewardship. Clin Infect Dis. 2007;44:159-177.
29. Davey P, Steinke D, MacDonald T, et al. Not so simple cystitis: how should prescribers be supported to make informed decisions about the increasing prevalence of infections caused by drug-resistant bacteria? Br J Gen Prac. 2000;50:143-146.

30. Health Protection Agency. Management of infection guidance for primary care consultation and local adaptation. 2010. Available at: http://www.hpa.org.uk/web/HPAwebfile/HPAweb_C/1279888711402. Accessed May 3, 2011

31. Sundqvist M, Kahlmeter G. 'Pre-emptive culturing' will improve the chance of 'getting it right' when empirical therapy of urinary tract infection fails. J Antimicrob Chemother. 2009;64:227-228.

32. Grover ML, Bracamonte JD, Kanodia AK, et al. Urinary tract infection in women over the age of 65: is age alone a marker of complication? J Am Board Fam Med. 2009;22:266-271.

33. Warren WJ, Abrutyn E, Hebel JR, et al. Guidelines for antimicrobial treatment of uncomplicated acute bacterial cystitis and acute pyelonephritis in women. Clin Infect Dis. 1999;29:745-758.

34. Hooton TM, Besser R, Foxman B, et al. Acute uncomplicated cystitis in an era of increasing antibiotics resistance: a proposed approach to empirical therapy. Clin Infect Dis. 2004;39:75-80.

35. Lutters M, Vogt-Ferrier NB. Antibiotic duration for treating uncomplicated, symptomatic lower urinary tract infections in elderly women. Cochrane Database Syst Rev. 2008;3:CD001535.

36. Loeb M, Brazil K, Lohfeld L, et al. Effect of a multifaceted intervention on number of antimicrobial prescriptions for suspected urinary tract infections in residents of nursing homes: cluster randomised controlled trial. BMJ. 2005;331:669.

37. Little P, Moore MV, Turner S, et al. Effectiveness of five different approaches in management of urinary tract infection: randomised controlled trial. BMJ. 2010;340:c199.

38. Howell AB, Vorsa N, Der MA, Foo LY. Inhibition of the adherence of P-fimbriated Escherichia coli to uroepithelial-cell surfaces by proanthocyanidin extracts from cranberries. N Engl J Med. 1998;339: $1085-1086$.

39. Jepson RG, Craig JC. Cranberries for preventing urinary tract infections. Cochrane Database Syst Rev. 2009;1:CD001321.

40. McMurdo MET, Bissett LY, Price RJ, et al. Does ingestion of cranberry juice reduce symptomatic urinary tract infections in older people in hospital? A double-blind, placebo-controlled trial. Age Ageing. 2005;34: 256-261.

41. Committee on Safety of Medicines and the Medicines and Healthcare Products Regulatory Agency. Interactions between warfarin and cranberry juice: new advice. MHRA/CSM current problems in pharmacovigilance. 2004;30:10. Available at: http://www.mhra.gov. uk/Howweregulate/Medicines/Herbalmedicines/HerbalSafetyNews/ Currentsafetyissues/CON1004343. Accessed May 3, 2011.

42. Mellen CK, Ford M, Rindone JP. Effect of high-dose cranberry juice on the pharmacodynamics of warfarin in patients. Br J Clin Pharmacol. 2010;70:139-142.

43. Niël-Weise BS, van den Broek PJ. Urinary catheter policies for shortterm bladder drainage in adults. Cochrane Database Syst Rev. 2005; 3:CD004203.
Clinical Interventions in Aging

\section{Publish your work in this journal}

Clinical Interventions in Aging is an international, peer-reviewed journal focusing on evidence-based reports on the value or lack thereof of treatments intended to prevent or delay the onset of maladaptive correlates of aging in human beings. This journal is indexed on PubMed Central, MedLine, the American Chemical Society's 'Chemical Abstracts Ser-

\section{Dovepress}

vice' (CAS), Scopus and the Elsevier Bibliographic databases. The manuscript management system is completely online and includes a very quick and fair peer-review system, which is all easy to use. Visit http://www.dovepress.com/testimonials.php to read real quotes from published authors. 